\title{
Characterization of phenolic compounds from normal ginger (Zingiber officinale Rosc.) and black ginger (Kaempferia parviflora Wall.) using UPLC-DAD-QToF-MS
}

\author{
Gelila Asamenew ${ }^{1} \cdot$ Heon-Woong Kim ${ }^{1} \cdot$ Min-Ki Lee $^{1} \cdot$ Seon-Hye Lee ${ }^{1}$ Young Jin Kim ${ }^{1} \cdot$ Youn-Soo Cha ${ }^{2}$. \\ Seon Mi Yoo ${ }^{1}$. Jung-Bong Kim ${ }^{1}$ (i)
}

Received: 16 August 2018 / Revised: 18 October 2018 / Accepted: 27 October 2018 / Published online: 20 November 2018

(C) The Author(s) 2018

\begin{abstract}
Zingiberaceae is the large diverse family comprised of rhizomatous plants with a higher concentration of phenolic compounds. The normal ginger (Zingiber officinale Rosc.) and black ginger (Kaempferia parviflora Wall.) belongs to this family and have similar morphological characteristics of rhizome, but their phenolic composition revealed differently. On this study, the phenolic composition of normal ginger (from Ethiopia and Korea) and black gingers (from Korea and Thailand) were profiled using ultra performance liquid chromatography coupled with diode array detector, quadrupole time-of-flight mass spectrometry (UPLC-DAD-QToF-MS). The result revealed that gingerol-related phenolic acid was detected only in normal gingers while, the methoxyflavones were identified exclusively in black gingers. 6-gingerol, 8-gingerol, 10-gingerol, 1-dehydro-6-gingerdione, and diacetoxy-8-gingerdiol were the major constituents among 18 phenolic acids detected from normal gingers, and 3,5,7,3',4'-pentamethoxyflavone and 5,7,4'-trimethoxyflavone were confirmed as predominant constituents among 13 methoxyflavones from black gingers. The total phenolic content was 434.7 and $698.1 \mathrm{mg} / 100 \mathrm{~g}$ dry weight (DW) in Korean and Ethiopian sample, respectively. The total methoxyflavone concentration was $4388.0 \mathrm{mg} / 100 \mathrm{~g}$ DW and $3940.2 \mathrm{mg} / 100 \mathrm{~g}$ DW in Korean and Thai black ginger sample, respectively. The higher concentration of methoxyflavones is from advanced biosynthetic pathway related to accumulated color of black ginger. In addition, new possible structural fragmentation was proposed for gingerol homologous series as additional pathway under the positive ionization mode, and further rationalized that ferulic acid moiety is the biosynthetic precursor. The result suggested that despite the morphological similarity, normal and black ginger showed distinct biosynthetic difference to synthesis their major secondary metabolites.
\end{abstract}

Electronic supplementary material The online version of this article (https://doi.org/10.1007/s00217-018-3188-z) contains supplementary material, which is available to authorized users.

Jung-Bong Kim

jungbkim@korea.kr

1 National Institute of Agricultural Science, Rural Development Administration, 166, Nongsaengmyeong-ro, Iseo-myeon, Wanju-gun, Jeollabuk-do, Republic of Korea

2 Department of Food Science and Human Nutrition, Chonbuk National University, 567 Baekje-daero, deokjin-gu, Jeonju-si, Jeollabuk-do, Republic of Korea 


\section{Graphical Abstract}

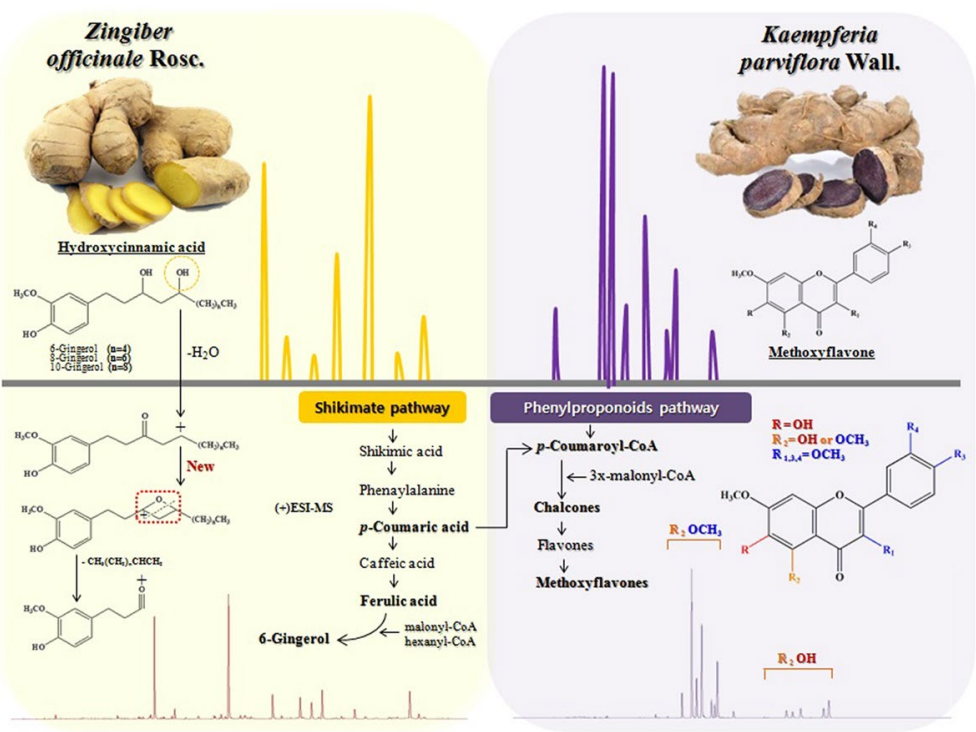

Keywords Kaempferia parviflora Wall. $\cdot$ Phenolic compounds $\cdot$ UPLC-DAD-QToF-MS · Zingiberaceae $\cdot$ Zingiber officinale Rosc.

\section{Introduction}

The Zingiberaceae family comprises about 53 genera and a total of over 1200 species [1]. It is widely distributed in the tropical region of Asia, Africa, and America. These plants are taxonomically characterized as perennial, aromatic, tuberous and non-tuberous rhizomes [2]. The rhizome is the main part of the plant which is economically important and a rich source of effective phytoconstituents for biological activities [2,3].

Ginger (Zingiber officinale Rosc.) is one of the most popular species belonging to this family. China, Thailand, Vietnam, India, and Indonesia are considered to be center of origin. It has been mainly utilized as a food seasoning ingredient and medicinal resource particularly for treating diseases related to inflammation and oxidative stress [4-8]. For its wide range of health functionality, the presence of higher concentration of phenolic derivatives plays an important role [5, 8-10].

Over the last three decades, extensive studies have been conducted to understand the detailed chemical composition and biological activities of normal ginger. Phenolic acids, diarylheptanoids, terpenoids, and flavonoids were reported to exist in ginger rhizomes [10-19]. In Supplementary Table 1, comprehensive library containing a total of 72 gingerols and diarylheptanoids derivatives from ginger rhizomes is presented as summary. Gingerol- and shogaolrelated derivatives are the principal medicinally active components contributing to the characteristic pungent flavor of ginger together with essential oil major component, zingerone $[19,20]$. The maturation state, environment, cultivar, and processing steps are major factors to influence the biosynthesis and concentration of the chemical composition in the rhizomes [21].

Black ginger (Kaempferia parviflora Wall.), Kra-chaiDum (in Thai), is another species with deep purple-colored rhizomes under the Zingiberaceae family, and shares a morphological resemblance with normal ginger. Thailand is recognized as a center of diversity, and distribution to different region of Asia [22]. In previous studies, 25 methoxyflavones and their glycosides were identified and quantified as predominant flavonoids from rhizome extracts (Supplementary Table 2) [23-26]. The presence of these compounds contributed to a wider range of medicinal effects such as antiinflammatory [27], antioxidant [28, 29], antiproliferative activity [30], hepatoprotective effect [31], and anticancer [32].

In Ethiopia, the history of ginger cultivation and utilization dates back from thirteenth century, and it is one of the spices contributing significantly to the country economy [33]. However, regardless of the significant cultivation, the status of phenolic constituents and its contribution to product quality standardization remains unstudied. Also, no studies have been reported on phenolic composition comparisons between normal and black ginger. Hence, in this study, the phenolic composition of gingers cultivated in 
Ethiopia, Korea, and Thailand were profiled in reference to constructed LC-MS libraries. Furthermore, the different phytochemical biosynthesis between normal and black ginger were considered.

The aims of the present study were to characterize the phenolic compounds of the normal and black ginger using ultra performance liquid chromatography coupled with diode array detector, quadrupole time-of-flight mass spectrometry (UPLC-DAD-QToF-MS), and to conduct quantitative comparison between the test samples.

\section{Materials and methods}

\section{Chemicals}

Acetonitrile, methanol, and water (HPLC-grade) were obtained from Fisher Scientific (Fair Lawn, NJ, USA). Formic acid was purchased from Junsei Chemical (Tokyo, Japan). 6-gingerol, 8-gingerol, 10-gingerol, 6-shogaol, 8-shogaol, and 10-shogaol were used as pure standards for identification. Galangin and 2,4,5-trimethoxycinnamic acid were used as internal standard. All standard chemicals were purchased from Sigma Aldrich Co. (St. Louis, MO, USA).

\section{Plant material}

Four ginger rhizome samples (each two samples of Zingiber officinale Rosc. and Kaempferia parviflora Wall.) were obtained in 2017. The voucher specimens were deposited in the Department of Agro-food Resources, National Institute of Agricultural Sciences, Rural Development Administration (Korea). Korean ginger (RDAZO117) was purchased from the local market (Wanju province, Korea). Ethiopian ginger (variety name; Bozia mau32/b 79, RDAZO217) was obtained from the local market (Ziway city, Ethiopia). Black ginger (RDAKP117) was obtained from Jeju Island (Korea). Thai black ginger (RDAKP217) was purchased from NEXTOBE co. LTD (Thailand). All ginger samples were lyophilized, pulverized and stored below $-60{ }^{\circ} \mathrm{C}$ prior to analysis.

\section{Extraction of phenolic acids and methoxyflavones}

Sample extraction was conducted according to the method described by Kim et al. [34] with minimum modification. The powdered rhizomes $(1 \mathrm{~g})$ were mixed with $10 \mathrm{~mL}$ of extraction solvent (methanol: water: formic acid $=80: 15: 5$, $\mathrm{v} / \mathrm{v} / \mathrm{v}$ ) containing $250 \mathrm{ppm}$ of 2,4,5-trimethoxycinnamic acid as internal standard. The mixture was vortexed, stirred with an orbital shaker for $5 \mathrm{~min}$ at $200 \mathrm{rpm}$ and centrifuged for $15 \mathrm{~min}$ at $3000 \mathrm{rpm}, 10^{\circ} \mathrm{C}$. The supernatant was filtered using syringe filter $(0.45 \mu \mathrm{m}$, PVDF, Whatman, Kent, England). $0.5 \mathrm{~mL}$ of the filtrate was diluted with water to $5 \mathrm{~mL}$ of final volume. The crude phenolic extract was then isolated by solid phase extraction method using Sep-Pak $\mathrm{C}_{18}$ cartridge (Waters Co., Milford, MA, USA). The cartridge was activated by washing with $2 \mathrm{~mL}$ of methanol, followed by $2 \mathrm{~mL}$ of water for conditioning. Then the diluted phenolic extract was loaded in to the cartridge, and impurities were removed by washing with $2 \mathrm{~mL}$ of water. Finally, the crude phenolic acid was eluted from the cartridge using $3 \mathrm{~mL}$ of methanol. The purified extract was concentrated using $\mathrm{N}_{2}$ gas, and then re-dissolved with $0.2 \mathrm{~mL}$ of the extract solvents without internal standard prior to instrumental analysis. For identification of methoxyflavones from black ginger, $1 \mathrm{~g}$ of powdered sample was taken and extracted with solvent system (methanol: water: formic acid $=50: 45: 5$, $\mathrm{v} / \mathrm{v} / \mathrm{v}$ ) containing $100 \mathrm{ppm}$ of galangin as an internal standard. The extraction and purification steps were followed as described above. All experimental analyses were performed in triplicates.

\section{UPLC-DAD-QToF-MS analysis}

Ultra performance liquid chromatography with photodiode array detector (Waters Co., Milford, MA, USA) coupled with quadrupole time-of-flight mass spectrometry (Waters Micromass, Manchester, UK) was used for analysis. UV spectra were taken in the region of $210-400 \mathrm{~nm}$ (representative wavelengths of $280 \mathrm{~nm}, 350 \mathrm{~nm}$ for phenolic acid and methoxyflavone, respectively). Chromatographic condition was conducted: column, Cortexs ${ }^{\circledR} \mathrm{UPLC}^{\circledR} \mathrm{T} 31.6 \mu \mathrm{m} \mathrm{C} 18$, $2.1 \mathrm{~mm} \times 150 \mathrm{~mm}$ (Waters Co.); pre-column, Security Guard ULTRA Cartridges, UHPLC C18 for 2.1 ID column (Phenomenex, Torrance, CA, USA); oven temperature $30^{\circ} \mathrm{C}$; for phenolic acid analysis, mobile phase was used $0.1 \%$ formic acid in water $(A)$ and $0.1 \%$ formic acid in acetonitrile $(B)$; flow rate $0.30 \mathrm{~mL} / \mathrm{min}$ (the elution gradient used as follow: 0-15 min, 5\% B; $15 \mathrm{~min}, 35 \%$ B; $20 \mathrm{~min}, 50 \%$ B; 35-40 min, $75 \% \mathrm{~B} ; 45-50 \mathrm{~min}, 5 \% \mathrm{~B})$. For methoxyflavone analysis, $0.5 \%$ formic acid in water $(A)$ and $0.5 \%$ formic acid in acetonitrile $(B)$; flow rate $0.30 \mathrm{~mL} / \mathrm{min}$ (the elution gradient used as follow: $0-15 \mathrm{~min}, 5 \% \mathrm{~B} ; 15 \mathrm{~min}, 38 \% \mathrm{~B} ; 30 \mathrm{~min}$, $50 \% \mathrm{~B} ; 35-40 \mathrm{~min}, 80 \% \mathrm{~B} ; 45-50 \mathrm{~min}, 5 \% \mathrm{~B})$. The mass spectrometric settings used were: ion source temperature $140{ }^{\circ} \mathrm{C}$, desolvation temperature $500{ }^{\circ} \mathrm{C}$, desolvation gas $1020 \mathrm{~L} \mathrm{~h}^{-1}$, capillary voltage $3.5 \mathrm{kV}$, and sampling cone voltage $40 \mathrm{~V}$. Mass analysis was run in positive ion mode using an electrospray ionization (ESI) source, and their range measured $50-1000 \mathrm{~m} / \mathrm{z}$ in full scan mode.

\section{Identification and quantification of phenolic compounds}

The identification of phenolic compounds was conducted for the pure standard available, by comparing their UV and 
mass spectral data. The rest of the peaks were determined by comparing retention time, UV spectra, and mass fragmentation presented in the library constructed from previous literatures (Supplementary Tables 1 and 2). The quantification was performed using internal standard without considering relative response factor.

\section{Statistical analysis}

The data analysis was performed by SPSS statistical data analysis software (PASW statistic 18). All data expressed as a mean \pm standard error of the mean (SEM). Data were considered statistically significant at a value of $p<0.05$.

\section{Results and discussion}

Several analytical approaches were developed to identify phytochemicals and authenticating ginger samples from possible contaminating plant material having similar appearance with ginger (adulterants) [12]. High-performance liquid chromatography coupled with electrospray ionization tandem mass spectrometry (HPLC-ESI-MS/MS) has shown better sensitivity for identification of compounds from ginger rhizomes [11, 13, 19, 21]. Recently, with the current analytical technology advancement, UPLC-DAD-QToF-MS becomes a powerful tool in providing excellent sensitivity and resolution for determination of natural products. Moreover, it provides best separation capability in higher throughput and time saving to run complex sample matrix compared to HPLC-ESI-MS/MS [35]. With this merit, in the current study, we identified phenolic compounds from normal and black ginger samples using UPLC-DAD-QToF-MS.

The phenolic acids and methoxyflavones characterized in the normal and black gingers are presented in Tables 1 and 2, respectively, including UV spectra, mass fragmentation $(\mathrm{m} / \mathrm{z})$, and contents. Especially, the positive ionization mode was used to obtain clear mass fragmentation as well as sodium $\left(\mathrm{Na}^{+}, m / z 23\right)$ and potassium $\left(\mathrm{K}^{+}, m / z 39\right)$ adducts ion which helps to confirm the parent ion of the peak unambiguously [11, 36]. Gingerol-related phenolic acid derivatives (wavelength at $280 \mathrm{~nm}$ ) were identified only from normal ginger, while black ginger showed different spectral peaks on UPLC-DAD chromatograms (Figs. 1, 3).

\section{Characterization of phenolic acids from normal gingers}

Figure 1 showed that peaks 3, 7, and 13 were eluted at retention time of 19.4, 23.3, and 27.7 min with high intensity at $280 \mathrm{~nm}$. These peaks, respectively, were differentiated by $28 \mathrm{Da}\left(\mathrm{CH}_{2}-\mathrm{CH}_{2}\right.$ moiety) for their corresponding $[\mathrm{M}+\mathrm{Na}]^{+},\left[\mathrm{M}+\mathrm{H}-\mathrm{H}_{2} \mathrm{O}\right]^{+}$, and $\left[\mathrm{M}+\mathrm{H}-2 \mathrm{H}_{2} \mathrm{O}\right]^{+}$. Actually, fragment ions at $\mathrm{m} / \mathrm{z} 259.1943,287.2295$, and 315.2657 were observed after losing of $2 \mathrm{H}_{2} \mathrm{O}$ from the parent ions as well as constant patterns of $\mathrm{m} / \mathrm{z} 179.0899,177.1153$, 145.0795, 137.0734, 117.0813, and 115.0669 from each peak. In the previous study, Jiang et al. [11] proposed the fragmentation scheme of gingerol-related derivatives. In peak 3, the predominant ion $(\mathrm{m} / \mathrm{z}$ 177.1153) was formed due to the loss of a neutral alkyl moiety $\left[\mathrm{CH}_{3}\left(\mathrm{CH}_{2}\right)_{4} \mathrm{CHO}, 100\right.$ Da] from rearranged structure of $\left[\mathrm{M}+\mathrm{H}-\mathrm{H}_{2} \mathrm{O}\right]^{+}$, and further fragmented by loss of $\mathrm{CHCHCH}_{2}(40 \mathrm{Da})$ to form $\mathrm{m} / \mathrm{z}$ 137.0734 (Scheme 1b). However, as shown in Fig. 2a, the $\mathrm{m} / \mathrm{z}$ 179.0899, 145.0795, and 117.0813 ions observed could not be rationalized from this fragmentation scheme. This suggested that peak 3 have another additional fragmentation pathway under positive ionization mode. From gingerolrelated structures $(\mathrm{n}=4,6,8)$, product ions at $\mathrm{m} / z 179.0899$ were formed with the remained ketone group after cleavage of bond at $\left.\mathrm{C} 3-\mathrm{C} 4\left[-\mathrm{CH}_{3}\left(\mathrm{CH}_{2}\right)_{n} \mathrm{CHCH}_{3}\right)\right]$ (Scheme 1a) instead of cleavage of bond at $\mathrm{C} 4-\mathrm{C} 5$ with the neutral alkyl chains $\left.\left[-\mathrm{CH}_{3}\left(\mathrm{CH}_{2}\right)_{n} \mathrm{CHO}\right)\right]$ (Scheme $1 \mathrm{~b}$ ), and eventually produces the predominant ion at $m / z 177.1153$ by the loss of $2 \mathrm{H}$ from $\mathrm{C} 2-\mathrm{C} 3$ position. As the biosynthesis precursor for gingerols derivatives, it was reported that ferulic acid produced intense peak at $\mathrm{m} / \mathrm{z}, 177$ after the removal of $\mathrm{H}_{2} \mathrm{O}$ from the protonated ion ([ferulic acid $+\mathrm{H}]^{+}, m / z$ 195), these ions showed subsequent losses of $\mathrm{CH}_{3} \mathrm{OH}$ and $\mathrm{CH}_{3} \mathrm{OH}-\mathrm{CO}$ to yielded the fragment ions at $\mathrm{m} / \mathrm{z}, 145$ and 117 , respectively [36]. Based on these proposed fragmentation, the additional accurate ions at $\mathrm{m} / \mathrm{z} 145.0795$ and 117.0813 actually resulted from subsequent loss of methoxyl group and -CO from 177.1153 ions, respectively (Scheme 1a). Therefore, the higher abundance of fragment ion at $\mathrm{m} / \mathrm{z} 177.1153$ was observed from the two fragmentation pathways. In the previous literature review, this type of fragmentation behavior for gingerol derivatives was not discussed hence we propose possible fragmentation scheme for the first time in our report. Finally, by comparing the MS data with pure standards, peak 3, 7 and 13 were identified as 6-gingerol, 8 -gingerol, and 10-gingerol according to the fragmentation presented above, respectively.

Peak 4 eluted at $21.38 \mathrm{~min}$ and showed similar fragmentation pattern to 6-gingerol with methylene $\left(\mathrm{CH}_{2}\right.$, $14 \mathrm{Da}$ ) increment on the fragment ion, which indicated that it is a methylated derivative of 6-gingerol. The position of methylation was confirmed by fragment ions at $\mathrm{m} / \mathrm{z} 191.1283\left(\left[\mathrm{M}+\mathrm{H}-\mathrm{H}_{2} \mathrm{O}-\mathrm{CH}_{3}\left(\mathrm{CH}_{2}\right)_{4} \mathrm{CHO}\right]^{+}\right.$or $\left.\left[\mathrm{M}+\mathrm{H}-\mathrm{H}_{2} \mathrm{O}-\mathrm{CH}_{3}\left(\mathrm{CH}_{2}\right)_{4} \mathrm{CHCH}_{3}\right]^{+}\right)$and 151.0897 $\left[\mathrm{M}+\mathrm{H}-\mathrm{H}_{2} \mathrm{O}-\mathrm{CH}_{3}\left(\mathrm{CH}_{2}\right)_{4} \mathrm{CHO}-\mathrm{CHCHCH}_{2}\right]^{+}$due to methyl substitution in the phenyl moiety. Therefore, peak 4 tentatively identified as methyl-6-gingerol by comparing the observation with the literature $[11,14,37]$.

In peak 8 (23.41 min) with a small amount, the $\mathrm{m} / \mathrm{z} 319.3065$ and 259.1956 ions were found through 
Table 1 Peak assignment and quantitative data of phenolic acids presented in normal ginger samples

\begin{tabular}{|c|c|c|c|c|c|c|c|c|c|}
\hline Peak no. & Compound name & $\mathrm{RT}(\min )$ & $\mathrm{UV} \lambda_{\max }(\mathrm{nm})$ & $\begin{array}{l}\text { Molecular } \\
\text { formula }\end{array}$ & $\begin{array}{l}\text { Theo- } \\
\text { retical }(\mathrm{m} / \mathrm{z}) \\
{[\mathrm{M}+\mathrm{H}]^{+}} \\
{[\mathrm{M}+\mathrm{Na}]^{+}}\end{array}$ & $\begin{array}{l}\text { Experimen- } \\
\operatorname{tal}(\mathrm{m} / \mathrm{z}) \\
{[\mathrm{M}+\mathrm{H}]^{+}} \\
{[\mathrm{M}+\mathrm{Na}]^{+}}\end{array}$ & $\begin{array}{l}\text { Fragment ions } \\
(\mathrm{m} / \mathrm{z})\end{array}$ & Korea & Ethiopia \\
\hline 1 & $\begin{array}{l}\text { 5-Acetoxy- } \\
\text { 1,7-bis(4- } \\
\text { hydroxy-3-meth- } \\
\text { oxyphenyl) } \\
\text { heptan-3-one }\end{array}$ & 18.56 & 220,280 & $\mathrm{C}_{23} \mathrm{H}_{28} \mathrm{O}_{7}$ & $\begin{array}{l}417.1919 \\
439.1738\end{array}$ & $\begin{array}{l}417.2328 \\
\text { ND }\end{array}$ & $\begin{array}{l}357.21,259.22, \\
219.19,179.09, \\
153.07\end{array}$ & $2.13 \pm 0.01$ & $2.22 \pm 0.02$ \\
\hline 2 & 6-Gingerdiol & 18.73 & 220,280 & $\mathrm{C}_{17} \mathrm{H}_{28} \mathrm{O}_{4}$ & $\begin{array}{l}297.2071 \\
319.1891\end{array}$ & $\begin{array}{l}\text { ND } \\
319.2247\end{array}$ & $\begin{array}{c}261.21,177.11 \\
163.09,137.07\end{array}$ & $14.74 \pm 0.17$ & $11.31 \pm 0.22$ \\
\hline $3^{\mathrm{a}}$ & 6-Gingerol & 19.40 & 231,280 & $\mathrm{C}_{17} \mathrm{H}_{26} \mathrm{O}_{4}$ & $\begin{array}{l}295.1915 \\
317.1734\end{array}$ & $\begin{array}{l}\text { ND } \\
317.1999\end{array}$ & $\begin{array}{l}277.21,259.19, \\
179.09,177.12, \\
145.08,135.07 \\
117.08,115.07\end{array}$ & $200.07 \pm 1.00$ & $376.40 \pm 0.40$ \\
\hline 4 & Methyl-6-gingerol & 21.38 & 229,279 & $\mathrm{C}_{18} \mathrm{H}_{28} \mathrm{O}_{4}$ & $\begin{array}{l}309.2071 \\
331.1891\end{array}$ & $\begin{array}{l}309.1454 \\
331.2185\end{array}$ & $\begin{array}{c}291.23,273.21 \\
191.13,151.09\end{array}$ & $3.65 \pm 0.02$ & $4.86 \pm 0.01$ \\
\hline $5^{\mathrm{b}}$ & $\begin{array}{l}\text { 3-Acetoxy- } \\
\text { 6-gingerdiol/5- } \\
\text { acetoxy-6-ging- } \\
\text { erdiol }\end{array}$ & 21.63 & 229,272 & $\mathrm{C}_{19} \mathrm{H}_{30} \mathrm{O}_{5}$ & $\begin{array}{l}339.2177 \\
361.1994\end{array}$ & $\begin{array}{l}\mathrm{ND} \\
361.2366\end{array}$ & $\begin{array}{c}321.24,261.21 \\
163.09,137.07\end{array}$ & $2.15 \pm 0.02$ & $1.25 \pm 0.05$ \\
\hline 6 & $\begin{array}{l}\text { Diacetoxy-4-ging- } \\
\text { erdiol }\end{array}$ & 22.20 & 269,281 & $\mathrm{C}_{19} \mathrm{H}_{28} \mathrm{O}_{6}$ & $\begin{array}{l}353.1970 \\
375.1789\end{array}$ & $\begin{array}{l}\text { ND } \\
375.2180\end{array}$ & $\begin{array}{c}\text { 293.21, 233.18, } \\
163.09,137.07\end{array}$ & $0.68 \pm 0.00$ & $0.84 \pm 0.01$ \\
\hline $7^{\mathrm{a}}$ & 8-Gingerol & 23.31 & 221,281 & $\mathrm{C}_{19} \mathrm{H}_{30} \mathrm{O}_{4}$ & $\begin{array}{l}323.2228 \\
345.2047\end{array}$ & $\begin{array}{l}323.2299 \\
345.2388\end{array}$ & $\begin{array}{l}305.24,287.23, \\
179.09,177.12, \\
145.08,117.02, \\
115.07\end{array}$ & $52.99 \pm 0.12$ & $70.35 \pm 0.34$ \\
\hline 8 & Acetoxy-6-gingerol & 23.41 & 220,281 & $\mathrm{C}_{19} \mathrm{H}_{28} \mathrm{O}_{5}$ & $\begin{array}{l}337.2020 \\
359.1840\end{array}$ & $\begin{array}{l}\text { ND } \\
359.2207\end{array}$ & $\begin{array}{l}319.31,259.20 \\
137.08\end{array}$ & - & - \\
\hline $9^{\mathrm{b}}$ & $\begin{array}{l}\text { Methyl-3-acetoxy- } \\
\text { 6-gingerdiol/ } \\
\text { methyl-5-ace- } \\
\text { toxy-6-gingerdiol }\end{array}$ & 23.74 & 225,279 & $\mathrm{C}_{20} \mathrm{H}_{32} \mathrm{O}_{5}$ & $\begin{array}{l}353.2333 \\
375.2153\end{array}$ & $\begin{array}{l}\text { ND } \\
375.2538\end{array}$ & $\begin{array}{c}335.26,275.22 \\
191.13,151.09\end{array}$ & $1.28 \pm 0.01$ & ND \\
\hline $10^{\mathrm{a}}$ & 6-Shogaol & 24.12 & 226,281 & $\mathrm{C}_{17} \mathrm{H}_{24} \mathrm{O}_{3}$ & $\begin{array}{l}277.1809 \\
299.1629\end{array}$ & $\begin{array}{l}277.2034 \\
\text { ND }\end{array}$ & 137.08 & $2.66 \pm 0.02$ & $9.10 \pm 0.04$ \\
\hline 11 & $\begin{array}{l}\text { Diacetoxy-6-ging- } \\
\text { erdiol }\end{array}$ & 26.08 & 227,281 & $\mathrm{C}_{21} \mathrm{H}_{32} \mathrm{O}_{6}$ & $\begin{array}{l}381.2283 \\
403.2102\end{array}$ & $\begin{array}{l}\text { ND } \\
403.2566\end{array}$ & $\begin{array}{l}321.24,261.21, \\
177.11,163.09, \\
137.07\end{array}$ & $8.63 \pm 0.04^{n s}$ & $8.36 \pm 0.15^{n s}$ \\
\hline 12 & $\begin{array}{l}\text { 1-Dehydro-6-ging- } \\
\text { erdione }\end{array}$ & 26.78 & $220,254,371$ & $\mathrm{C}_{17} \mathrm{H}_{22} \mathrm{O}_{4}$ & $\begin{array}{l}291.1602 \\
313.1421\end{array}$ & $\begin{array}{l}291.2058 \\
\text { ND }\end{array}$ & $177.07,149.08$ & $18.12 \pm 0.10$ & $55.72 \pm 0.19$ \\
\hline $13^{\mathrm{a}}$ & 10-Gingerol & 27.72 & 220,281 & $\mathrm{C}_{21} \mathrm{H}_{34} \mathrm{O}_{4}$ & $\begin{array}{l}351.2541 \\
373.2360\end{array}$ & $\begin{array}{l}\text { ND } \\
373.2749\end{array}$ & $\begin{array}{l}333.28,315.26 \\
179.09,177.12, \\
145.08,137.08 \\
117.08,115.07\end{array}$ & $83.48 \pm 0.05$ & $96.08 \pm 0.33$ \\
\hline 14 & $\begin{array}{l}\text { Methyl diacetoxy- } \\
\text { 6-gingerdiol }\end{array}$ & 28.68 & 229,279 & $\mathrm{C}_{22} \mathrm{H}_{34} \mathrm{O}_{6}$ & $\begin{array}{l}395.2430 \\
417.2259\end{array}$ & $\begin{array}{l}\text { ND } \\
417.2716\end{array}$ & $\begin{array}{l}412.31,335.26, \\
275.23,177.12, \\
151.09\end{array}$ & $1.52 \pm 0.01$ & $0.32 \pm 0.01$ \\
\hline 15 & $\begin{array}{l}\text { Diacetoxy-8-ging- } \\
\text { erdiol }\end{array}$ & 30.61 & 220,278 & $\mathrm{C}_{23} \mathrm{H}_{36} \mathrm{O}_{6}$ & $\begin{array}{l}409.2596 \\
431.2415\end{array}$ & $\begin{array}{l}\text { ND } \\
431.2866\end{array}$ & $\begin{array}{c}349.28,289.25 \\
177.11,137.07\end{array}$ & $21.23 \pm 0.11$ & $34.76 \pm 0.24$ \\
\hline 16 & $\begin{array}{l}\text { 1-Dehydro-8-ging- } \\
\text { erdione }\end{array}$ & 31.57 & $220,253,370$ & $\mathrm{C}_{19} \mathrm{H}_{26} \mathrm{O}_{4}$ & $\begin{array}{l}319.1914 \\
341.1734\end{array}$ & $\begin{array}{l}319.2307 \\
\text { ND }\end{array}$ & 177.11 & $3.96 \pm 0.07$ & $8.61 \pm 0.20$ \\
\hline 17 & $\begin{array}{l}\text { Acetoxy-10-gin- } \\
\text { gerol }\end{array}$ & 32.43 & 227,281 & $\mathrm{C}_{23} \mathrm{H}_{36} \mathrm{O}_{5}$ & $\begin{array}{l}393.2646 \\
415.2466\end{array}$ & $\begin{array}{l}\text { ND } \\
415.2923\end{array}$ & $\begin{array}{c}333.28,315.26 \\
177.11,137.08\end{array}$ & $6.73 \pm 0.04$ & ND \\
\hline 18 & $\begin{array}{l}\text { 1-Dehydro-10-gin- } \\
\text { gerdione }\end{array}$ & 36.31 & $220,253,370$ & $\mathrm{C}_{21} \mathrm{H}_{30} \mathrm{O}_{4}$ & $\begin{array}{l}347.2228 \\
369.2047\end{array}$ & $\begin{array}{l}347.2707 \\
\text { ND }\end{array}$ & $177.11,149.09$ & $10.65 \pm 0.10$ & $17.93 \pm 0.18$ \\
\hline Total & & & & & & & & $434.67 \pm 0.22$ & $698.11 \pm 0.13$ \\
\hline
\end{tabular}

All samples analyzed in positive ionization mode, ND; Not detected, (-) below quantification level, $[\mathrm{M}+\mathrm{Na}]^{+}$and $[\mathrm{M}+\mathrm{H}]^{+}$actual mass provided, $\left({ }^{a}\right)$ further confirmed in comparison with authentic standards, $\left({ }^{b}\right)$ exact position of the acetoxy substitute not confirmed, each value calculated as mean \pm standard error of mean (SEM) of three replicates, content expressed in $\mathrm{mg} / 100 \mathrm{~g}$ DW; dry weight, $\left({ }^{n s}\right.$ ) compound showed no significant difference between the two sample means $(p>0.05)$ 
Table 2 Peak assignment and quantitative data of methoxyflavones presented in black ginger sample

\begin{tabular}{|c|c|c|c|c|c|c|c|c|c|}
\hline $\begin{array}{l}\text { Peak } \\
\text { no. }\end{array}$ & Compound name & $\begin{array}{l}\text { RT } \\
(\min )\end{array}$ & $\mathrm{UV} \lambda_{\max }(\mathrm{nm})$ & $\begin{array}{l}\text { Molecular } \\
\text { formula }\end{array}$ & $\begin{array}{l}\text { Theoreti- } \\
\text { cal }(m / z) \\
{[\mathrm{M}+\mathrm{H}]^{+}}\end{array}$ & $\begin{array}{l}\text { Experi- } \\
\text { mental } \\
(\mathrm{m} / \mathrm{z}) \\
{[\mathrm{M}+\mathrm{H}]^{+}}\end{array}$ & $\begin{array}{l}\text { Fragment } \\
\text { ions } \\
(\mathrm{m} / \mathrm{z})\end{array}$ & Korea & Thailand \\
\hline $1^{\mathrm{a}}$ & $\begin{array}{l}\text { 6-Hydroxy-7,4'- } \\
\text { Dimethoxyflavone }\end{array}$ & 14.23 & $229,264,330$ & $\mathrm{C}_{17} \mathrm{H}_{14} \mathrm{O}_{5}$ & 299.0925 & 299.1125 & $\begin{array}{l}337.05 \\
321.07\end{array}$ & $24.46 \pm 0.69$ & $21.60 \pm 0.59$ \\
\hline 2 & $\begin{array}{l}5,7,3^{\prime}, 4^{\prime} \text {-Tetrameth- } \\
\text { oxyflavone (tetra- } \\
\text { methylluteolin) }\end{array}$ & 16.86 & $242,265,335$ & $\mathrm{C}_{19} \mathrm{H}_{18} \mathrm{O}_{6}$ & 343.1187 & 343.1519 & $\begin{array}{l}381.07 \\
365.10\end{array}$ & $209.56 \pm 5.42$ & $174.73 \pm 5.15$ \\
\hline 3 & $\begin{array}{l}3,5,7,3^{\prime}, 4^{\prime} \text {-Pentameth- } \\
\text { oxyflavone (pen- } \\
\text { tamethaquercetin) }\end{array}$ & 17.82 & 249,339 & $\mathrm{C}_{20} \mathrm{H}_{20} \mathrm{O}_{7}$ & 373.1293 & 373.1803 & $\begin{array}{l}411.09 \\
395.11\end{array}$ & $1333.70 \pm 18.05^{n s}$ & $1262.70 \pm 10.40^{n s}$ \\
\hline 4 & 5,7-Dimethoxyflavone & 18.32 & 263,307 & $\mathrm{C}_{17} \mathrm{H}_{14} \mathrm{O}_{4}$ & 283.0976 & 283.1452 & $\begin{array}{l}321.056 \\
305.08 \\
268.09\end{array}$ & $385.26 \pm 10.63$ & $312.00 \pm 8.78$ \\
\hline 5 & $\begin{array}{l}\text { 5,7,4'-Trimethoxyfla- } \\
\text { vone (trimethylapi- } \\
\text { genin) }\end{array}$ & 18.82 & $230,264,326$ & $\mathrm{C}_{18} \mathrm{H}_{16} \mathrm{O}_{5}$ & 313.1081 & 313.1637 & $\begin{array}{l}351.07 \\
335.09 \\
298.10\end{array}$ & $975.31 \pm 19.86^{n s}$ & $908.06 \pm 5.23^{n s}$ \\
\hline 6 & $\begin{array}{l}\text { 3,5,7-Trimethoxyfla- } \\
\text { vone }\end{array}$ & 20.12 & 262,326 & $\mathrm{C}_{18} \mathrm{H}_{16} \mathrm{O}_{5}$ & 313.1081 & 313.1533 & $\begin{array}{l}351.07 \\
335.09\end{array}$ & $140.08 \pm 4.00$ & $121.20 \pm 3.47$ \\
\hline 7 & $\begin{array}{l}3,5,7,4^{\prime} \text {-Tetramethoxy- } \\
\text { flavone (tetramethyl- } \\
\text { kaempferol) }\end{array}$ & 20.38 & $233,264,336$ & $\mathrm{C}_{19} \mathrm{H}_{18} \mathrm{O}_{6}$ & 343.1187 & 343.1695 & $\begin{array}{l}381.08 \\
365.11 \\
328.10\end{array}$ & $574.50 \pm 15.17^{n s}$ & $559.99 \pm 4.01^{n s}$ \\
\hline $8^{b}$ & $\begin{array}{l}\text { 5-Hydroxy-7,3', } 4^{\prime}- \\
\text { trimethoxyflavone }\end{array}$ & 25.11 & $231,267,343$ & $\mathrm{C}_{18} \mathrm{H}_{16} \mathrm{O}_{6}$ & 329.1031 & 329.1066 & $\begin{array}{l}351.07 \\
314.08\end{array}$ & $8.78 \pm 0.20$ & $4.82 \pm 0.09$ \\
\hline $9^{b}$ & $\begin{array}{l}\text { 5-Hydroxy-3,7,3',4'- } \\
\text { tetramethoxyflavone } \\
\text { (ayanin) }\end{array}$ & 27.29 & $253,270,353$ & $\mathrm{C}_{19} \mathrm{H}_{18} \mathrm{O}_{7}$ & 359.1136 & 359.1324 & $\begin{array}{l}381.10 \\
344.09 \\
329.07\end{array}$ & $110.39 \pm 2.77$ & $74.45 \pm 1.84$ \\
\hline $10^{\mathrm{b}, \mathrm{c}}$ & $\begin{array}{l}\text { 5-Hydroxy-7-methox- } \\
\text { yfavone (tectochry- } \\
\text { sin) }\end{array}$ & 27.90 & $225,267,310$ & $\mathrm{C}_{16} \mathrm{H}_{12} \mathrm{O}_{4}$ & 269.0819 & 269.1070 & 254.06 & $95.95 \pm 2.46$ & $71.84 \pm 1.80$ \\
\hline $11^{\mathrm{b}, \mathrm{c}}$ & $\begin{array}{l}\text { 5-Hydroxy-7,4'- } \\
\text { dimethoxyfalvone }\end{array}$ & 28.70 & $231,267,331$ & $\mathrm{C}_{17} \mathrm{H}_{14} \mathrm{O}_{4}$ & 299.0925 & 299.1150 & 284.07 & $151.52 \pm 3.39$ & $114.73 \pm 2.73$ \\
\hline $12^{\mathrm{b}, \mathrm{c}}$ & $\begin{array}{l}\text { 5-Hydroxy- } \\
\text { 3,7-dimethoxyfla- } \\
\text { vone }\end{array}$ & 30.99 & $227,265,310,350$ & $\mathrm{C}_{17} \mathrm{H}_{14} \mathrm{O}_{5}$ & 299.0925 & 299.1190 & 284.07 & $147.71 \pm 6.82$ & $116.14 \pm 3.13$ \\
\hline $13^{\mathrm{b}}$ & $\begin{array}{l}\text { 5-Hydroxy-3,7,4'- } \\
\text { trimethoxyflavone }\end{array}$ & 31.51 & $232,267,347$ & $\mathrm{C}_{18} \mathrm{H}_{16} \mathrm{O}_{6}$ & 329.1031 & 329.1277 & $\begin{array}{l}351.09 \\
314.08\end{array}$ & $230.82 \pm 13.86$ & $197.99 \pm 4.25$ \\
\hline Total & & & & & & & & $4388.04 \pm 103.32$ & $3940.24 \pm 51.46$ \\
\hline
\end{tabular}

All samples analyzed in positive ionization mode, $\left({ }^{\mathrm{a}}\right)$ tentatively identified, $\left({ }^{\mathrm{b}}\right)[\mathrm{M}+\mathrm{K}]^{+}$not provided, $\left({ }^{\mathrm{c}}\right)[\mathrm{M}+\mathrm{Na}]^{+}$not provided, fragment ions $(\mathrm{m} / \mathrm{z})$ of $\left[\mathrm{M}+\mathrm{H}-\mathrm{CH}_{3}\right]^{+}$and $\left[\mathrm{M}+\mathrm{H}-2 \mathrm{CH}_{3}\right]^{+}$are presented. Each value calculated as mean \pm standard error of mean (SEM) of three replicates, content expressed in $\mathrm{mg} / 100 \mathrm{~g}$ DW; dry weight, $\left({ }^{n s}\right)$ compound showed no significant difference between the two sample means $(p>0.05)$

successive losses of $\mathrm{H}_{2} \mathrm{O}$ and $\mathrm{AcOH}(60 \mathrm{Da})$ from parent ion. The base peak at $\mathrm{m} / \mathrm{z} 137.0750$ was produced by cleavage of alkyl chain including acetoxy group. Based on observed fragmentation, peak 8 was identified as acetoxy6-gingerol [11, 13, 14]. Also, peak 17 showed characteristic fragmentation pattern similar to peak 8 with $56 \mathrm{Da}$ $\left(\mathrm{CH}_{2}-\mathrm{CH}_{2}-\mathrm{CH}_{2}-\mathrm{CH}_{2}\right)$ increment on its fragment ions at $\mathrm{m} / \mathrm{z} 333.2766[\mathrm{M}+\mathrm{H}-\mathrm{AcOH}]^{+}$, hence this peak tentatively identified as acetoxy-10-gingerol.

Peak 2 (6-gingerdiol, $\mathrm{m} / \mathrm{z}$ 319) was differentiated from 6-gingerol $(\mathrm{m} / \mathrm{z}, 317)$ with a difference of $2 \mathrm{Da}$ at $[\mathrm{M}+\mathrm{Na}]^{+}$, and intense peak at $\mathrm{m} / z, 261.2138$ was formed by the loss of $2 \mathrm{H}_{2} \mathrm{O}$ from two hydroxyl groups on $\mathrm{C} 3$ and C5 positions of parent ion [14, 19] (Fig. 2b). As minor compound, peak 5 (21.63 $\mathrm{min}$ ) tentatively identified as 3-acetoxy-6-gingerdiol or 5-acetoxy-6-gingerdiol by fragment ions at $\mathrm{m} / \mathrm{z} 321.2396$ and 261.2122 corresponding to the losses of $\mathrm{H}_{2} \mathrm{O}$ and $\mathrm{AcOH}$ (60 Da), respectively, from gingerdiol structure. Furthermore, as methylated derivatives of acetoxy-6-gingerdiol, methyl-3- or methyl-5-acetoxy-6-gingerdiol (peak 9, $23.74 \mathrm{~min}$ ) were presumed by fragment ions at $m / z 335.2615$ and 275.2209 with methylene $\left(\mathrm{CH}_{2}, 14 \mathrm{Da}\right)$ increment from peak 5. Additionally, product ions at $\mathrm{m} / z 191.1309$ and 151.0915 evidenced that 


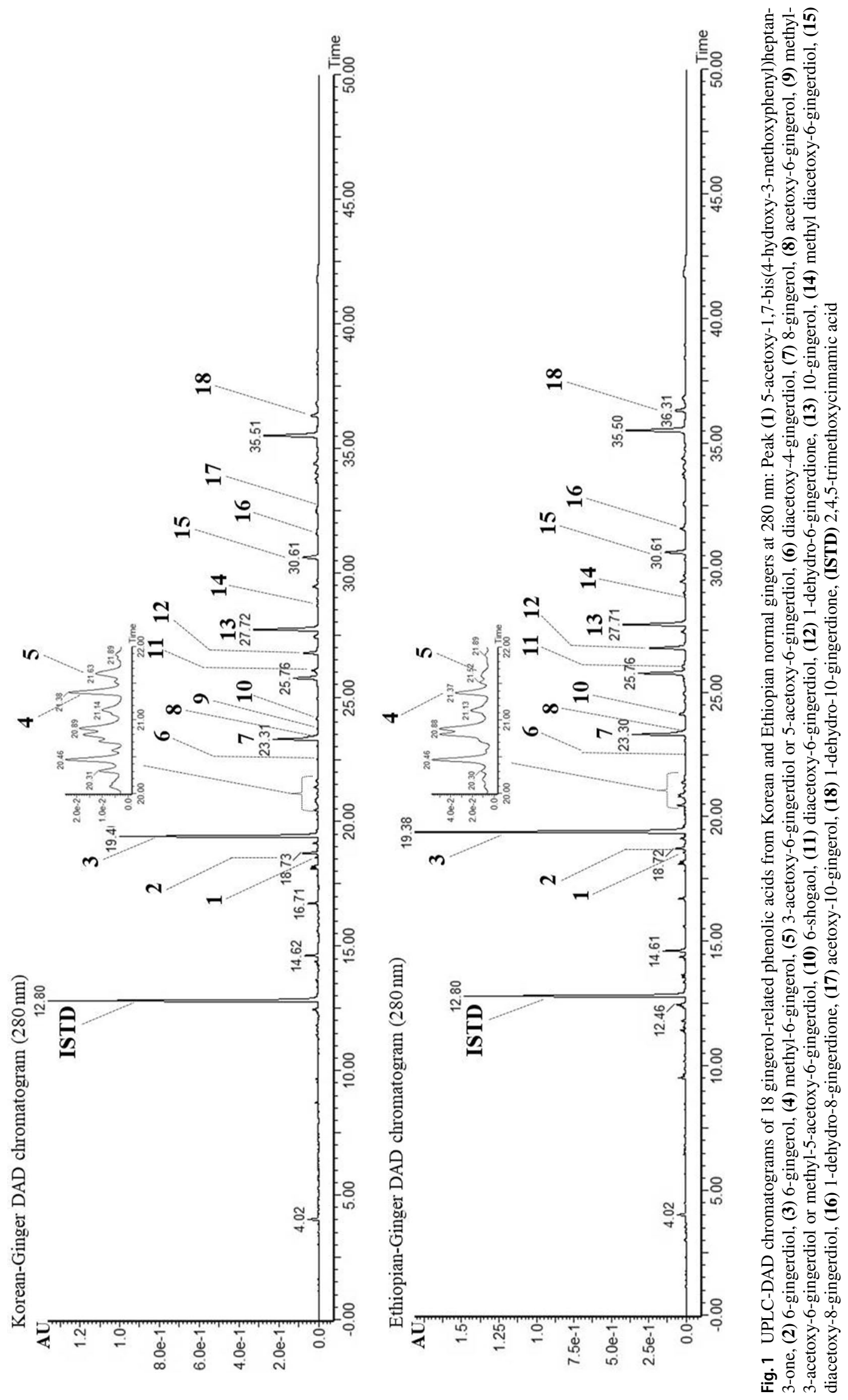




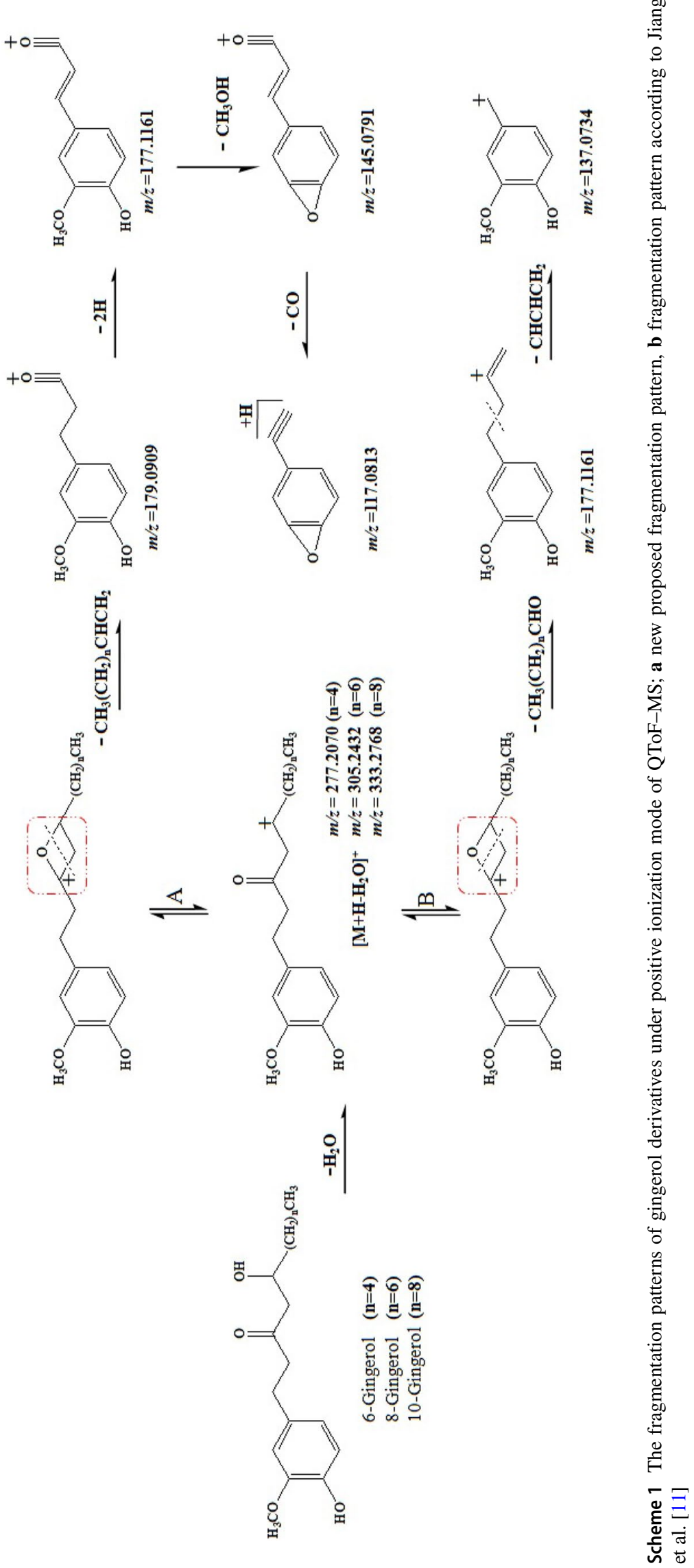



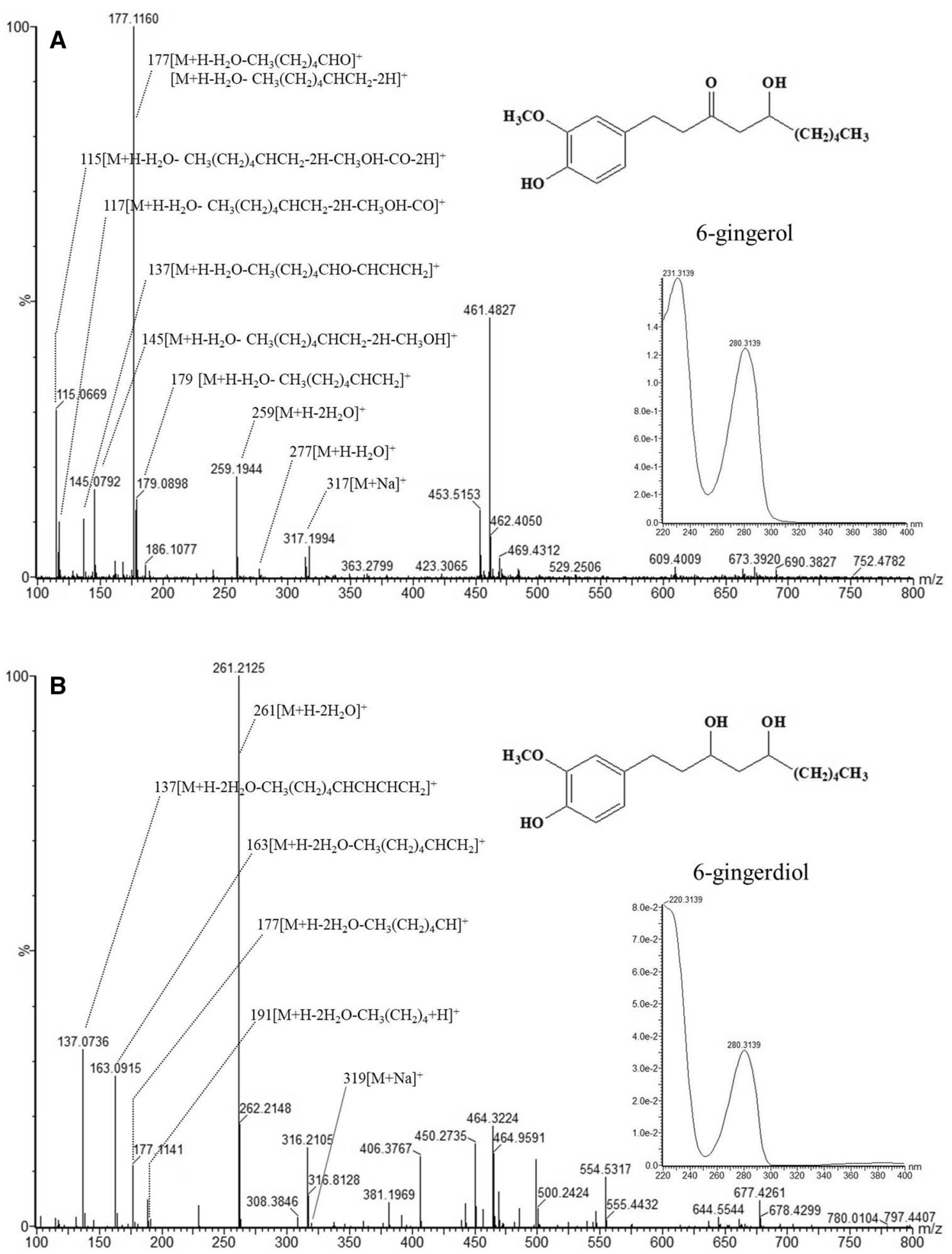

Fig. 2 (+) ESI-MS and UV spectra of 6-gingerol (a) and 6-gingerdiol (b) from normal gingers

this compound further methylated on the phenyl position like methyl-6-gingerol (peak 4) [11, 14]. The exact position of the acetoxy substitute needs to be further confirmed from acetoxy-6-gingerdiol-related phenolics (peak 5 and 9) by NMR technique.

The formation of fragment ions at $\mathrm{m} / \mathrm{z}, 321.2404$ $[\mathrm{M}+\mathrm{H}-\mathrm{AcOH}]^{+}$and $261.2115[\mathrm{M}+\mathrm{H}-2 \mathrm{AcOH}]^{+}$with the 

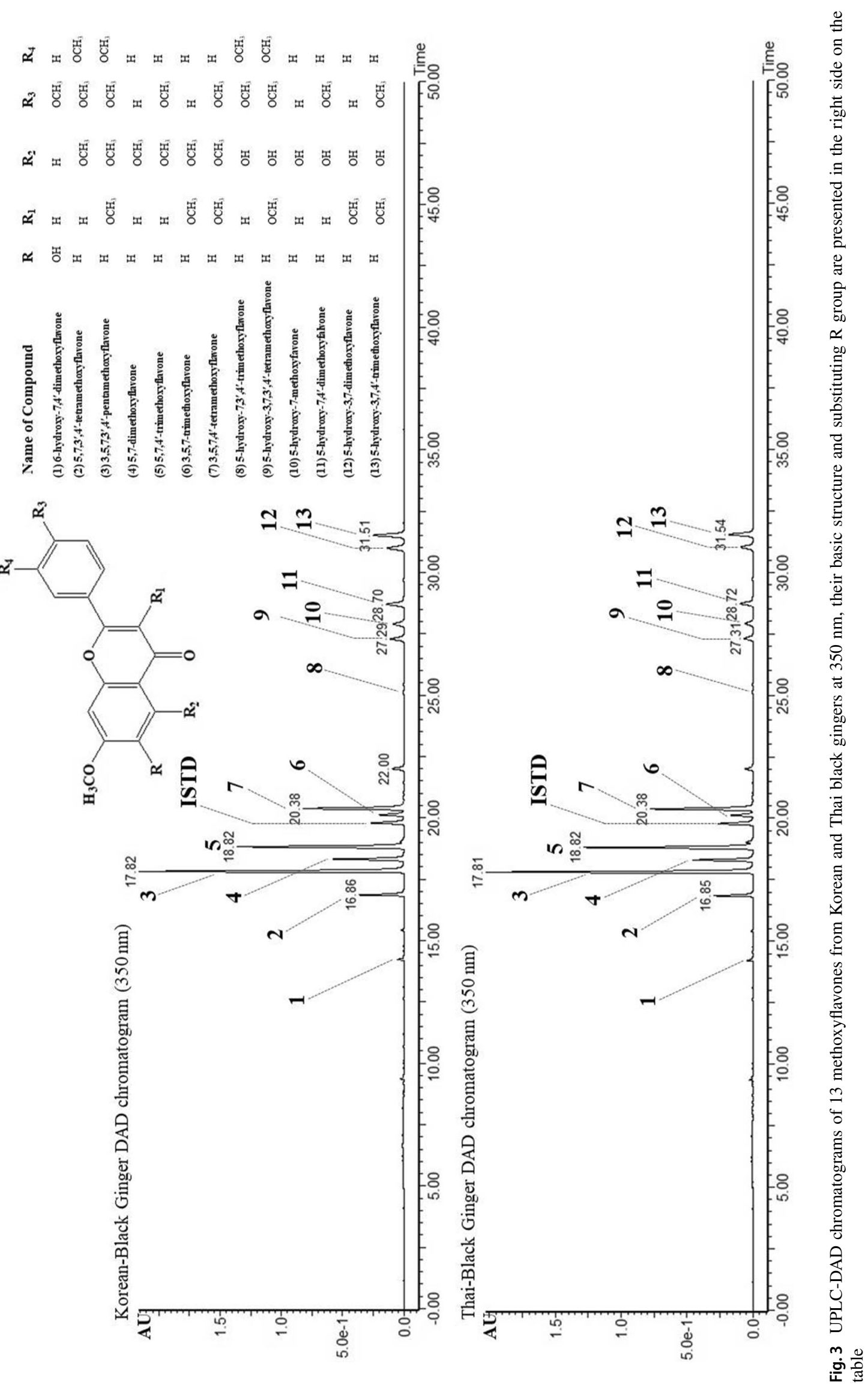
literature comparison suggested that peak 11 is di-acetylated derivative of 6-gingerdiol with low absorbance (UV spectra at $280 \mathrm{~nm}$ ) compared to 6-gingerol (Fig. 2; Table 1). This could also be confirmed as gingerdiol structure through the absence of ketone group on the aliphatic chain. Therefore, peak 11 identified unambiguously as diacetoxy-6-gingerdiol [14]. Peaks 6 and 15 showed similar fragmentation pattern in reference to diacetoxy-6-gingerdiol. They were tentatively identified as diacetoxy-4-gingerdiol and diacetoxy-8-gingerdiol by decrease $(375.2175,293.2072$, and 233.1781) and increase (431.2866, 349.2791, and 289.2470) of $\mathrm{CH}_{2}-\mathrm{CH}_{2}(28 \mathrm{Da})$ for adduct and fragment ions, respectively, corresponding to $[\mathrm{M}+\mathrm{Na}]^{+},[\mathrm{M}+\mathrm{H}-\mathrm{AcOH}]^{+}$, and $[\mathrm{M}+\mathrm{H}-2 \mathrm{AcOH}]^{+}$from diacetoxy-6-gingerdiol. Peak 14 (28.68 $\mathrm{min}$ ) showed similar fragmentation pattern to diacetoxy-6-gingerdiol with methylene $\left(\mathrm{CH}_{2}, 14 \mathrm{Da}\right)$ increment on the fragment ions, therefore, it was identified as methyl diacetoxy-6-gingerdiol [11].

Gingerdione-related compounds showed similar UV spectra at 220,253, and $370 \mathrm{~nm}$ with fragment ions of $[\mathrm{M}+\mathrm{H}]^{+}$ and $\left[\mathrm{M}+\mathrm{H}-\mathrm{CH}_{3}\left(\mathrm{CH}_{2}\right)_{n} \mathrm{OCHCH}_{2}, n=4,6,8\right]^{+}$by $\mathrm{CH}_{2}-\mathrm{CH}_{2}$ (28 Da) increments. Therefore, peaks 12, 16, and 18 were tentatively identified as 1-dehydro-6-gingerdione, 1-dehydro-8-gingerdione, and 1-dehydro-10-gingerdione, respectively [11, 14]. Also, peak 10 was identified as 6-shogaol with intense ion at $m / z 137.0762$ based on pure standard. Jolad et al. [14] reported that the characteristic fragment ion at $m / z 205$ was produced due to C5-C6 cleavage of the aliphatic chain on 6-shagaol, but it has not been observed in this study. In peak 1 , the characteristic fragmentation was confirmed from ions at $\mathrm{m} / \mathrm{z} 259.1819,219.1855$, and 179.0881 corresponding to diarylheptanoids under positive ionization mode [12]. Especially, the fragment ion at $\mathrm{m} / \mathrm{z}$ $357.2058[\mathrm{M}+\mathrm{H}-\mathrm{AcOH}]^{+}$suggested that this peak might be acetylated derivative of diarylheptanoid. Hence, peak 1 tentatively identified as 5-acetoxy-1,7-bis(4-hydroxy3-methoxyphenyl)heptan-3-one [17].

\section{Characterization of methoxyflavones from black gingers}

Methoxyflavones belong to a certain flavonoid class containing 1-7 methoxyl substitutes on the 2-phenyl chromone structure, and are predominantly distributed in the peel part of Citrus fruits [38]. In DAD chromatogram of Fig. 3, black ginger extracts showed different spectral patterns with maximum wavelength of $350 \mathrm{~nm}$ compared to normal ginger. Previous studies reported that methoxyflavones of aglycone type are the major chemical constituents in black ginger $[25,39]$. Also, several methoxyflavone glycosides including kaempferiaoside $\mathrm{A}, \mathrm{C}$, and $\mathrm{D}$ were additionally identified from this plant rhizome [24, 30, 31] (Supplementary Table 2). In the current study, a total of 13 methoxyflavone aglycones were clearly separated and identified from black ginger extracts collected from Jeju Island, Korea and Thailand (Fig. 3), and their names, UV spectra and adduct ions $\left([\mathrm{M}+\mathrm{K}]^{+},[\mathrm{M}+\mathrm{Na}]^{+}\right.$, and $\left.[\mathrm{M}+\mathrm{H}]^{+}\right)$are presented in Table 2 . The elution order of each peak was differentiated by number and position of adducted methoxyl group in the basic structure. In detail, peaks 2-3, 4-7, 8-9, and 10-13 were eluted slowly as the number of methoxyl group increased within their peak intervals (Fig. 3; Table 2). According to Xing et al. [38], methoxyflavones were generally fragmented by losing $n \mathrm{CH}_{3}, \mathrm{H}_{2} \mathrm{O}, \mathrm{CO}, \mathrm{H}_{2} \mathrm{O}+\mathrm{CH}_{3}, \mathrm{CO}+\mathrm{CH}_{3}$, and $\mathrm{CO}+\mathrm{H}_{2} \mathrm{O}+\mathrm{CH}_{3}$ from the parent ions under positive ionization mode, however, peaks 4,5 , and 7-13 formed only minor fragment ion after losing one $\mathrm{CH}_{3}$ from their parent ions in our study. Especially, peak 9 (5-hydroxy-3,7,3',4'tetramethoxyflavone, ayanin) further formed fragment ion at $m / z 329.0663$ corresponding to $\left[\mathrm{M}+\mathrm{H}-2 \mathrm{CH}_{3}\right]^{+}$. Finally, these methoxyflavones were not detected in normal ginger samples.

\section{Quantification of phenolic acid and methoxyflavone derivatives from gingers}

The contents of gingerol-related phenolics and methoxyflavones were calculated using 2,4,5-trimethoxycinnamic acid and galangin as internal standard, respectively. The total phenolic acid content presented on Table 1, Ethiopian ginger has a moderate higher concentration $(698.11 \mathrm{mg} / 100 \mathrm{~g}$, dry weight (DW)) than that of Korean ginger $(434.67 \mathrm{mg} / 100 \mathrm{~g}$, DW). Except diacetoxy-6-gingerdiol, the concentration of all identified compounds showed significant difference between two samples $(p<0.05)$. Especially, 6-gingerol identified as the major constituent with 46.03 and 53.92 proportions (\%) of the total content in Korean and Ethiopian ginger samples, respectively, followed by 10- and 8-gingerol. Previous studies by Jolad et al. [11], Jiang et al. [14], and He et al. [40] also supported that 6-gingerol was the major active compound in the rhizomes; however, according to Ahui et al. [37], 8-gingerol was confirmed as the major constituent from ginger of Nigeria origin. In addition, Zhan et al. [41] reported that $25.25 \mathrm{mg}$ of 10 -gingerol was obtained through gradient separation and purification from $100 \mathrm{mg}$ of crude extract. Hence, the representative major compound among samples is affected by several factors such as plant origin, environment, and state of rhizome maturity [13]. Moreover, this variation also further supported by a metabolomics approach on characterization of ginger samples collected from five different Asian countries (China, India, Malaysia, Thailand, and Vietnam) [21].

The contents of 1-dehydro-6-gingerdione and diacetoxy-8-gingerdiol were significantly higher next to gingerol homologous series. Acetoxy-6-gingerol was detected in both sample, however, its amount was below 
the quantification level. Methyl-3 or methyl-5-acetoxy6-gingerdiol and acetoxy-10-gingerol were not detected in Ethiopian ginger, and as minor compounds, the contents of 6-gingerdiol, 3-acetoxy or 5-acetoxy-6-gingerdiol and methyl diacetoxy-6-gingerdiol were slightly higher in Korean ginger. Also, the content of 1-dehydro-6-gingerdione $(55.72 \mathrm{mg} / 100 \mathrm{~g}$, DW) was significantly higher in Ethiopian sample (Table 1). This might be due to environmental factors to affect the biosynthesis and the concentration level of compounds from different origins. The proportion of 6 -shogaol accounts for only $1.14 \%$ of the total phenolic acid compared to 6-gingerol in Ethiopian sample. Shogaol is the dehydrated product of gingerol and this chemical change occurs in the process of thermal drying of the rhizomes and long-term storage [42]. In our treatment, the fresh samples were dried under lower temperature, hence, 6-shogaol was found to be small amount and the other shogaol derivatives were not detected.

3,5,7,3',4'-pentamethoxyflavone (PMF), 5,7,4'-trimethoxyflavone (5,7,4'-TMF), and 3,5,7,4'-tetramethoxyflavone (3,5,7,4'-TTMF) were presented as predominant aglycone among 13 methoxyflavones isolated from Korean and Thai black gingers (Table 2). Except PMF, 5,7,4'-TMF, and 3,5,7,4'-TTMF, all compounds were significantly different in concentration between both sample $(p<0.05)$. In total methoxyflavone contents, black ginger rhizomes of Korea (4388.04 mg/100 g, DW) and Thailand (3940.24 mg/100 g, DW) were much higher than the peels of Citrus reticulata (mandarin orange, 372.72-1547.49 mg/100 g, DW) and Citrus sinensis (orange, 110.05-259.07 mg/100 g, DW) reported [38]. Among 12 methoxyflavones determined from Thai black ginger, PMF (1347 mg/100 g, DW) was presented as the major compound, which is similar with our results (Korea, 1333.7; Thailand, $1262.7 \mathrm{mg} / 100 \mathrm{~g}$, DW) [24]. In the other study, PMF and 5,7,4'-TMF were also confirmed as the predominant methoxyflavones in black ginger [43].

The rhizomes of normal and black ginger are rich source of phenolic compounds, and very important biologically and economically due to the presences of these compounds. Our result confirmed that even though both plants are from the same family and have similar morphological characteristics, there is a distinct biosynthetic pathway to synthesize their major chemical constitutes. Hence, gingerol-related phenolics were only detected in normal ginger, while methoxyflavones were detected in black ginger. Especially, it was considered that black ginger is a major source of methoxyflavones with wide range of biological activities compared to other plants such as Citrus species.

Acknowledgements This study was carried out with the support of 'Cooperative Research Program for Agricultural Science and
Technology Development (Project No. PJ01178704)', National Institute of Agricultural Science, Rural Development Administration, Republic of Korea.

\section{Compliance with ethical standards}

Conflict of interest The authors declare that they have no competing interests.

Compliance with ethics requirements This article does not contain any studies with human or animal subjects.

Open Access This article is distributed under the terms of the Creative Commons Attribution 4.0 International License (http://creativeco mmons.org/licenses/by/4.0/), which permits unrestricted use, distribution, and reproduction in any medium, provided you give appropriate credit to the original author(s) and the source, provide a link to the Creative Commons license, and indicate if changes were made.

\section{References}

1. Kress WJ, Prince LM, Williams KJ (2002) The phylogeny and a new classification of the gingers (Zingiberaceae): evidence from molecular data. Am J Bot 89:1682-1696

2. Jatoi SA, Kikuchi A, Watanabe KN (2007) Genetic diversity, cytology, and systematic and phylogenetic studies in Zingiberaceae. G31:56-62

3. Ke J, Delin W, Te-lin W, Larsen K (2000) Zingiberaceae. Flora China 24:322-377

4. Gunathilake K, Rupasinghe HV (2015) Recent perspectives on the medicinal potential of ginger. Botanics 5:55-63

5. Peng F, Tao Q, Wu X, Dou H, Spencer S, Mang C, Xu L, Sun L, Zhao Y, Li H, Zeng S, Liu G, Hao X (2012) Cytotoxic, cytoprotective and antioxidant effects of isolated phenolic compounds from fresh ginger. Fitoterapia 83:568-585

6. Shareef HK, Muhammed HJ, Hussein HM, Hameed IH (2016) Antibacterial effect of ginger (Zingiber officinale Roscoe) and bioactive chemical analysis using gas chromatography-mass spectrometry. Oriental J Chem 32:817-837

7. Tohma H, İlhami Gülçin İ, Bursal E, Gören AC, Alwasel SH, Köksa E (2017) Antioxidant activity and phenolic compounds of ginger (Zingiber Officinale Rosc.) determined by HPLC-MS/MS. J Food Meas Char 11:556-566

8. Singh G, Kapoor IPS, Pratibha S, de Heluani CS, de Lampasona MP, Catalan CAN (2008) Chemistry, antioxidant and antimicrobial investigations on essential oil and oleoresins of Zingiber officinale. Food Chem Toxicol 46:3295-3302

9. Ali BH, Blunden G, Tanira MO, Nemmar A (2008) Some phytochemical, pharmacological and toxicological properties of ginger (Zingiber officinale Roscoe): A review of recent research. Food Chem Toxicol 46:409-420

10. Fathy MM, Eid HH, Hussein MA, Ahmed HH, Hussein AA (2015) The role of Zingiber officinale in the treatment of Alzheimer's disease: in-vitro and in-vivo evidence. Res J Pharm Biol Chem Sci 6:735-749

11. Jiang H, Solyom AM, Timmerman BN, Gang DR (2005) Characterization of gingerol-related compounds in ginger rhizome (Zingiber officinale Rosc.) by high-performance liquid chromatography/electrospray ionization mass spectrometry. Rapid Commun Mass Spectrom 19:2957-2964

12. Jiang H, Xie Z, Koo H, McLaughlin SP, Timmermann BN, Gang DR (2006) Metabolic profiling and phytogenetic analysis of 
medicinal Zingiber species: Tools for authentication of ginger (Zingiber officinale Rosc.). Phytochemistry 67:1673-1685

13. Jiang H, Timmermann BN, Gang DR (2007) Characterization and identification of diarylheptanoids in ginger (Zingiber officinale Rosc.) using high-performance liquid chromatography/electrospray ionization mass spectrometry. Rapid Commun Mass Spectrom 21:509-518

14. Jolad SD, Lantz CR, Solyom AM, Chen GJ, Timmermann BN (2004) Fresh organically grown ginger (Zingiber officinale): composition and effects on LPS-induced PGE2 production. Phytochemistry 65:1937-1954

15. Kikuzaki H, Nakatani N (1996) Cyclic diarylheptanoids from rhizomes of Zingiber officinale. Phytochemistry 43:273-277

16. Kikuzaki H, Usuguchi J, Nakatani N (1991) Constituents of Zingiberaceae. I. diarylheptanoids from the rhizomes of ginger (Zingiber officinale Roscoe). Chem. Pharm Bull 39:120-122

17. Ma J, Jin X, Yang L, Liu Z (2004) Diarylheptanoids from the rhizomes of Zingiber officinale. Phytochemistry 65:1137-1143

18. Mercy OA, Simeon OO, Saheed A, Ayokunle O, Temitope AE (2014) Analysis of phenolic compounds, phytosterols, lignans and stilbenoids in garlic and ginger oil by gas chromatography. J Food Chem Nutr 2:53-60

19. Tanaka K, Arita M, Sakurai H, Ono N, Tezuka Y (2015) Analysis of chemical properties of edible and medicinal ginger by metabolomics approach. BioMed Res Int. https://doi. org/10.1155/2015/671058

20. Shih H, Chern C, Kuo P, Wu Y, Chan Y, Liao Y, Cand T, Wu T (2014) Synthesis of analogs of gingerol and shogaol, the active pungent principles from the rhizomes of Zingiber officinale and evaluation of their anti-platelet aggregation effects. Int J Mol Sci 15:3926-3951

21. Yudthavorasit S, Wongravee K, Leepipatpiboon N (2014) Characteristic fingerprint based on gingerol derivative analysis for discrimination of ginger (Zingiber officinale) according to geographical origin using HPLC-DAD combined with chemometrics. Food Chem 158:101-111

22. Nopporncharoenkul N, Chanmai J, Jenjittikul T, AnamthawatJonsson K, Soontornchainaksaeng P (2017) Chromosome number variation and polyploidy in 19 Kaempferia (Zingiberaceae) taxa from Thailand and one species from Laos. J Syst Evol 55(5):466-476

23. Azuma T, Tanaka Y, Kikuzaki H (2008) Phenolic glycosides from Kaempferia parviflora. Phytochemistry 68:2743-2748

24. Ninomiya K, Matsumoto T, Chaipech S, Miyake S, Katsuyama Y, Tsuboyama A, Pongpiriyadacha Y, Hayakawa T, Muraoka O, Morikawa T (2016) Simultaneous quantitative analysis of 12 methoxyflavones with melanogenesis inhibitory activity from the rhizomes of Kaempferia parviflora. J Nat Med 70:179-189

25. Moon HI, Kim DW, Kim SK, Seo SH (2016) Effect of 6-hydroxy7,4'-dimethoxyflavone on antidiabetic effects in normal and streptozotocin-induced diabetic rats. Bulg Chem Commun 48:317-322

26. Chaipech S, Morikawa T, Ninomiya K, Yoshikawa M, Pongpiriyadacha Y, Hayakawa T, Muraoka O (2012) New flav-3-en-3-ol glycosides, kaempferiaosides $\mathrm{C}$ and $\mathrm{D}$, and acetophenone glycosides, kaempferiaosides E, and F, from the rhizomes of Kaempferia parviflora. J Nat Med 66:486-492

27. Horigome $S$, Yoshida I, Ito $S$, Inohana $S$, Fushimi K, Nagai $T$, Yamaguchi A, Fujita K, Satoyama T, Katsuda S, Suzuki S, Watai M, Hirose N, Mitsue T, Shirakawa H, Komai M (2014) Identification and evaluation of anti inflammatory compounds from Kaempferia parviflora. Biosci Biotechnol Biochem 78:851-860

28. Vichitphan S, Vichitphan K, Sirikhansaeng P (2007) Flavonoid content and antioxidant activity of Krachai-dum (Kaempferia parviflora) wine. KMITL J Sci Tech 7:97-105

29. Park J, Pyun H, Woo SW, Jeong J, Hwang J (2014) The protective effect of Kaempferia parviflora extracts on UVB- induced skin photo-aging in hairless mice. Photodermatol Photoimmunol Photomed 30:237-245
30. Wongsrikaew N, Kim H, Vichitphan K, Cho SK, Han J (2012) Antiproliferative activity and polymethoxyflavones composition analysis of Kaempferia parviflora extract. J Korean Soc Appl Biol Chem 55:813-817

31. Chaipech S, Morikawa T, Ninomiya K, Yoshikawa M, Pongpiriyadacha Y, Hayakawa T, Muraoka O (2012) Structures of two new phenolic glycosides, kaempferiaosides A and B, and hepatoprotective constituents from the rhizomes of Kaempferia parviflora. Chem Pharm Bull 60:62-69

32. Potikanond S, Sookkhee S, Na Takuathung M, Mungkornasawakul P, Wikan N, Smith DS, Nimlamool W (2017) Kaempferia parviflora extract exhibits anti-cancer activity against HeLa cervical cancer cells. Front Pharmacol 8:630. https://doi.org/10.3389/ fphar.2017.00630

33. Hegde V, Hegde S (2014) An economic overview of ginger production in Ethiopia. Int J Sci Res 3:2052-2054

34. Kim HW, Kim JB, Cho SM, Chung MN, Lee YM, Chu SM, Che JH, Kim SN, Kim SY, Cho YS, Kim JH, Park HJ, Lee DJ (2012) Anthocyanin changes in the Korean purple-fleshed sweet potato, Shinzami, as affected by steaming and baking. Food Chem 130:966-972

35. Churchwell MT, Twaddle NC, Meeker LR, Doerge DR (2005) Improving LC-MS sensitivity through increases in chromatographic performance: Comparisons of UPLC-ES/MS/MS to HPLC-ES/MS/MS. J Chromatogr B 825:134-143

36. Qi LW, Wen X, Cao J, Li C, Li P, Yi L, Wang Y, Cheng X, Ge $X$ (2008) Rapid and sensitive screening and characterization of phenolic acids, phthalides, saponins and isoflavonoids in Danggui buxиe Tang by rapid resolution liquid chromatography/diode array detection coupled with time-of-flight mass spectrometry. Rapid Commun Mass Spectrom 22:2493-2509

37. Ahui MLB, Konan AB, Zannou-Tchoko VJ, Amonkan AK, KatiCoulibaly S, Offoumou MA (2013) Identification of gingerols in ginger (Zingiber officinale Rosc.) by high-performance liquid chromatography-tandem mass spectrometry and pharmacologic studies of its aqueous extract on the rabbit isolated duodenum contractility. J Phys Pharm Adv 3:16-26

38. Xing TT, Zhao XJ, Zhang YD, Li YF (2017) Fast separation and sensitive quantitation of polymethoxylated flavonoids in the peels of Citrus using UPLC-Q-TOF-MS. J Agric Food Chem 65:2615-2627

39. Yorsin S, Kanokwiroon K, Radenahmad N, Jansakul C (2014) Effects of Kaempferia parviflora rhizomes dichloromethane extract on vascular functions in middle-aged male rat. J Ethnopharmacol 156:162-174

40. He X, Bernart MW, Lian L, Lin L (1998) High-performance liquid chromatography-electrospray mass spectrometric analysis of pungent constituents of ginger. J Chromatogr A 796:327-334

41. Zhan K, Xu K, Yin H (2011) Preparative separation and purification of gingerols from ginger (Zingiber officinale Roscoe) by high-speed counter-current chromatography. Food Chem 126:1959-1963

42. Balladin DA, Headley O (1998) High pressure liquid chromatographic analysis of the main pungent principles of solar dried West Indian ginger (Zingiber officinale Roscoe). Renew Energ 13:531-536

43. Burapan S, Kim M, Han J (2014) PMFs analysis of Krachaidum products by HPLC and GC. J Appl Biol Chem 57:211-218

44. Okabea Y, Shimada T, Horikawac T, Kinoshitaa K, Koyamaa K, Ichinose K, Aburada M, Takahashi K (2014) Suppression of adipocyte hypertrophy by polymethoxyflavonoids isolated from Kaempferia parviflora. Phytomedicine 21:800-806

45. Sae-Wonga C, Matsuda BH, Tewtrakul S, Tansakul P, Nakamura S, Nomura Y, Yoshikawa M (2011) Suppressive effects of methoxyflavonoids isolated from Kaempferia parviflora on inducible nitric oxide synthase (iNOS) expression in RAW 264.7 cells. J Ethnopharmacol 136:488-495 